\title{
New Pyrethrum Synergists from Dihydrodillapiole and Furapiole ${ }^{\dagger}$
}

\author{
S. K. Mukerjee, S. Walia, V. S. Saxena* and S. S. Tomar \\ Division of Agricultural Chemicals, Indian Agricultural \\ Research Institute, New Delhi-110012, India
}

Received November 4, 1981

\begin{abstract}
In order to discover more potent pyrethrum synergists, dihydrodillapiole and furapiole, two dillapiole-based compounds having considerable synergistic activity, were converted into various acyl and $\alpha, \beta$-unsaturated carbonyl derivatives. The synthesis, factor of synergism, spectroscopic data and structure activity relationships of these derivatives are reported.
\end{abstract}

Synergists play an important role in efficient, economic and practical formulations of pyrethrum. The synergistic activity of dillapiole (1-allyl -2,3-dimethoxy-4,5-methylenedioxy benzene), one of the major constituents $(27 \%$ ) of Indian dill (Anethum sowa Roxb.) seed oil, toward pyrethrum ${ }^{1)}$ and towards $N$-methyl carbamates has been reported earlier. ${ }^{2,3)}$ In an attempt to discover better synergists, a large number of derivatives of dillapiole have been synthesised by structural modifications. Amongst these, dihydrodillapiole (I) and furapiole (II) have shown considerably higher synergistic activity. ${ }^{4,5)}$ It was felt that further modifications of these two potent compounds may yield pyrethrum synergists of higher potency. In this paper the synthesis and synergistic activity of a large number of acyl derivatives of dihydrodillapiole and furapiole are reported. Further, an earlier work had shown that some $\alpha, \beta$-unsaturated carbonyl derivatives like 1-(2,3-dimethoxy-4,5methylenedioxyphenyl) non-1-en-3-one possess very high activity. ${ }^{4)}$ Attempts therefore have now been made to synthesize such compounds from the acyl derivatives of dihydrodillapiole and furapiole by condensation with different types of aldehydes.

\section{RESULTS AND DISCUSSION}

The acyl derivatives of both dihydrodillapiole and furapiole needed for this work were obtained by Friedel Craft's acylation in the presence of anhydrous zinc chloride in dry ether or dry methylene chloride. The formation of side products under these conditions was minimal. The yields, even under these conditions, were variable and not very good. The most important side reaction observed was the opening of the benzo-1,3-dioxole ring. This takes place with almost all the Lewis acids tried in these reactions and is very specific as reported earlier ${ }^{6)}$ with $\mathrm{BF}_{3}$-etherate, excluding its use in the present case. With anhydrous $\mathrm{AlCl}_{3}$, further complications due to demethylation were also observed.

None of the test chemicals showed any insecticidal activity up to the $1 \%$ level against Tribolium castaneum as the test insect. However, all of them showed a synergistic property toward pyrethrum. In the case of the acyl derivatives of dihydrodillapiole (III XII), their factors of synergism $\left(\mathrm{LC}_{50}\right.$ for

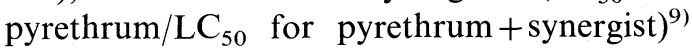
varied from 2.3 to 4.0 as compared to 2.1 for the standard reference synergist, piperonyl butoxide (PBO) and 2.3 for dihydrodillapiole (Table I).

† Contribution No. 198 from Division of Agricultural Chemicals, I.A.R.I., New Delhi-110012.

* Present address: Division of Entomology, IARI, New Delhi-110012, India. 
Table I. $R_{\mathrm{M}}$ Values and Factor of Synergism of

Various Test Chemicals

\begin{tabular}{|c|c|c|c|}
\hline $\begin{array}{c}\text { Structure } \\
\text { No. }\end{array}$ & Compound & $R_{\mathrm{M}}$ Value & $\begin{array}{l}\text { Factor of } \\
\text { synergism }\end{array}$ \\
\hline I & Dihydrodillapiole & - & 2.3 \\
\hline II & Furapiole & -0.13 & 5.2 \\
\hline III & Acetyldihydrodillapiole & -0.45 & 2.3 \\
\hline IV & Propionyl dihydrodillapiole & -0.31 & 2.5 \\
\hline $\mathbf{V}$ & Butyryl dihydrodillapiole & -0.16 & 2.7 \\
\hline VI & Valeryldihydrodillapiole & -0.04 & 3.1 \\
\hline VII & Caproyl dihydrodillapiole & +0.11 & 3.5 \\
\hline VIII & Heptanoyl dihydrodillapiole & +0.20 & 3.8 \\
\hline IX & Octanoyl dihydrodillapiole & +0.35 & 4.0 \\
\hline $\mathbf{X}$ & Pelargonyl dihydrodillapiole & +0.50 & 4.0 \\
\hline XI & Decanoyl dihydrodillapiole & +0.75 & 3.9 \\
\hline XII & Chloroacetyl dihydrodillapiole & -0.41 & 2.4 \\
\hline XIII & Acetyl furapiole & -0.50 & 3.2 \\
\hline XIV & Propionyl furapiole & -0.31 & 2.8 \\
\hline $\mathbf{X V}$ & Butyryl furapiole & -0.09 & 2.7 \\
\hline XVI & Heptanoyl furapiole & +0.20 & 1.6 \\
\hline XVII & Octanoyl furapiole & +0.35 & 1.6 \\
\hline XVIII & Decanoyl furapiole & +0.69 & 1.1 \\
\hline XIX & Chloroacetylfurapiole & -0.45 & 1.5 \\
\hline $\mathbf{X X}$ & Cinnamoyl dihydrodillapiole & -0.32 & 1.5 \\
\hline XXI & 3,4-Methylenedioxy cinnamoyl dihydrodillapiole & -0.45 & 1.0 \\
\hline XXII & 4-Nitrocinnamoyl dihydrodillapiole & -0.58 & - \\
\hline XXIII & 4-Chlorocinnamoyl dihydrodillapiole & -0.16 & 1.4 \\
\hline XXIV & 3-(2-Furyl propenoyl) dihydrodillapiole & -0.43 & 1.8 \\
\hline XXV & 2-Nonenoyl dihydrodillapiole & +0.60 & 4.5 \\
\hline XXVI & Cinnamoyl furapiole & -0.57 & 1.1 \\
\hline XXVII & 3,4-Methylenedioxy cinnamoyl furapiole & -0.75 & 1.0 \\
\hline XXVIII & 4-Nitrocinnamoyl furapiole & -0.69 & 1.2 \\
\hline XXIX & 4-Chlorocinnamoyl furapiole & -0.37 & 1.5 \\
\hline $\mathbf{X X X}$ & 3-(2-Furyl propenyl) furapiole & -0.65 & 2.1 \\
\hline XXXI & 3,4-Methylenedioxy cinnamoyl benzene & -0.37 & 1.5 \\
\hline XXXII & 2,3-Dimethoxy-4,5-methylenedioxy cinnamoyl benzene & -0.20 & 1.8 \\
\hline XXXIII & Piperonyl butoxide & - & 2.1 \\
\hline
\end{tabular}

The relationship between the synergistic activity of the acyl derivatives of dihydrodillapiole and their lipophilicity is shown in Fig. 1. The synergistic activity gradually increases with increasing length of the acyl chain up to $8 \sim 9$ carbon atoms and then decreases, but the lipophilicity continues to increase linearly. In this respect these derivatives behave the same way as the other derivatives examined earlier. ${ }^{5}$ On the other hand, the furapiole derivatives (XIII to XIX) show opposite behaviour. Thus, as seen in Fig. 2, although the lipophilicity of the acyl derivatives of furapiole increases with an increase in the number of carbon atoms, there is a steep fall in their synergistic activity.
This result is very surprising, as in all the cases examined earlier, synergistic activity increased with the lipophilicity of the molecule at least up to a few carbon atoms. The reason for this unexpected behaviour is not known yet.

$\alpha, \beta$-Unsaturated carbonyl compounds (XX $\sim \mathbf{X X X}$ ), derived from (III) or XIII and various aromatic aldehydes, exhibited very poor synergism compared with the starting acetyl derivatives. The introduction of nitro, chloro, and methylenedioxy groups in these compounds failed to improve the synergistic efficacy. In this series only one compound, non-2-enoyl dihydrodillapiole (XXV), showed synergism much superior to both the starting 


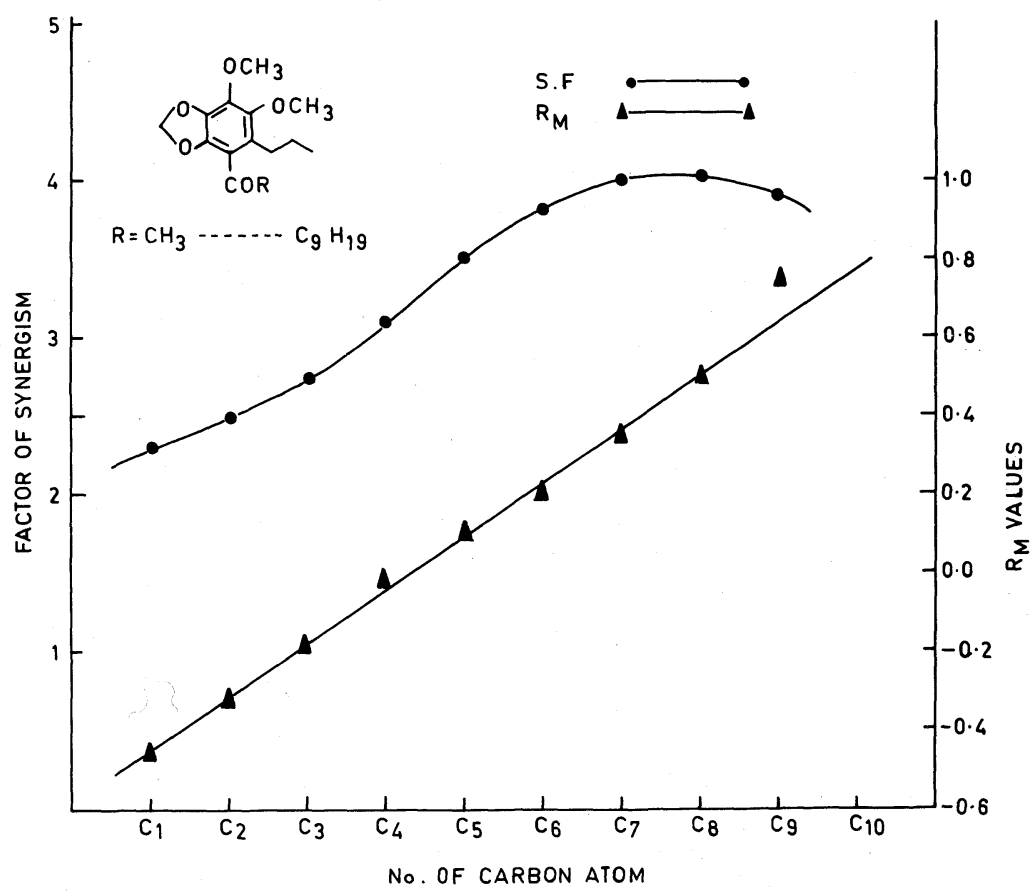

FIG. 1. Relationships between $R_{\mathrm{M}}$ Values, Factor of Synergism and Acyl Chain Length for Various Carbonyl Derivatives of Dihydrodillapiole.

$\mathbf{\Delta}-\mathbf{\Delta}, R_{\mathrm{M}}$ values; $-\mathbf{-}$, factor of synergism.

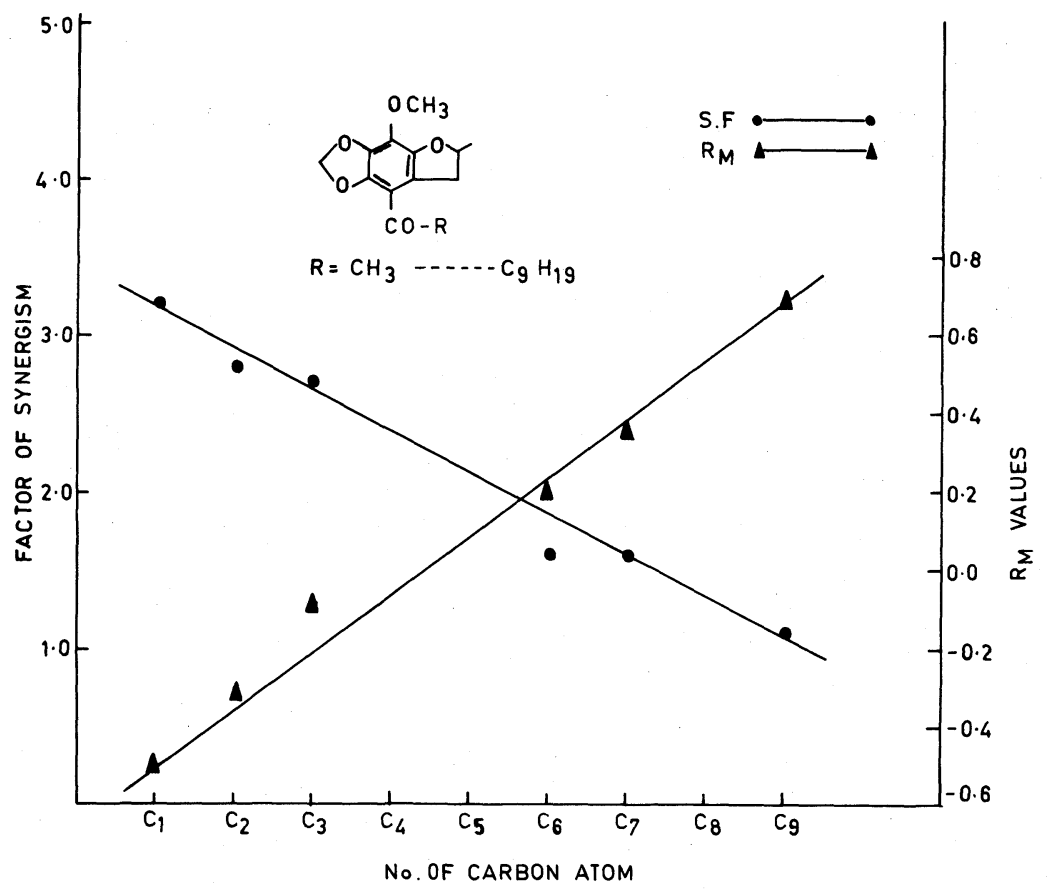

FIG. 2. Relationships between $R_{\mathrm{M}}$ Values, Factor of Synergism and Acyl Chain Length for Various Carbonyl Derivatives of Furapiole.

$\boldsymbol{\Delta}-\boldsymbol{\Delta}, R_{\mathrm{M}}$ values; $-\mathbf{O}$, factor of synergism. 


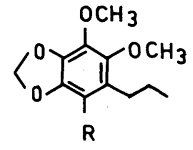

$\begin{array}{ll}\text { I } & R=H \\ \text { II } & R=\mathrm{COCH}_{3} \\ \text { IV } & R=\mathrm{COC}_{2} \mathrm{H}_{5} \\ \text { V } & R=\mathrm{COC}_{3} \mathrm{H}_{7} \\ \text { VI } & R=\mathrm{COC}_{4} \mathrm{Hg}_{9} \\ \text { VII } & R=\mathrm{COC}_{5} \mathrm{H}_{11} \\ \text { VIII } & R=\operatorname{COC}_{6} \mathrm{H}_{13} \\ \text { IX } & R=\operatorname{COC}_{7} \mathrm{H}_{15} \\ X & R=\mathrm{COC}_{8} \mathrm{H}_{17} \\ \text { XI } & R=\operatorname{COC}_{9} \mathrm{H}_{19} \\ \text { XII } & R=\mathrm{COCH}_{2} \mathrm{CI}\end{array}$

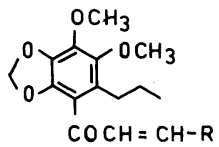

$\begin{array}{ll}\text { XX } & R=\mathrm{C}_{6} \mathrm{H}_{5} \\ \text { XXI } & R=\mathrm{C}_{7} \mathrm{H}_{5} \mathrm{O}_{2} \\ \text { XXII } & R=\mathrm{C}_{6} \mathrm{H}_{4} \mathrm{NO}_{2}(p) \\ \text { XXIII } & R=\mathrm{C}_{6} \mathrm{H}_{4} \mathrm{Cl}(p) \\ \text { XXIV } & R=\mathrm{C}_{4} \mathrm{H}_{3} \mathrm{O} \\ \text { XXV } & R=\mathrm{C}_{6} \mathrm{H}_{13}\end{array}$

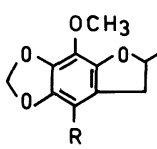

II $\quad \mathrm{R}=\mathrm{H}$

XIII $\quad \mathrm{R}=\mathrm{COCH}_{3}$

XIV $\quad \mathrm{R}=\mathrm{COC}_{2} \mathrm{H}_{5}$

$X V \quad R=\mathrm{COC}_{3} \mathrm{H}_{7}$

$X V I \quad R=\operatorname{COC}_{6} \mathrm{H}_{13}$

$X V I I \quad R=\mathrm{COC}_{7} \mathrm{H}_{15}$

$X V I I I \quad R \quad \mathrm{COC}_{9} \mathrm{H}_{19}$

XIX $R=\mathrm{COCH}_{2} \mathrm{Cl}$

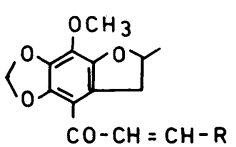

$X X V I \quad R=\mathrm{C}_{6} \mathrm{H}_{5}$

$X X V I I \quad R=\mathrm{C}_{7} \mathrm{H}_{5} \mathrm{O}_{2}$

XXVIII $\quad \mathrm{R}=\mathrm{C}_{6} \mathrm{H}_{4} \mathrm{NO}_{2}(p)$

XXIX $\quad \mathrm{R}=\mathrm{C}_{6} \mathrm{H}_{4} \mathrm{Cl}(p)$

$X X X \quad R=\mathrm{C}_{4} \mathrm{H}_{3} \mathrm{O}$<smiles>O=C(C=Cc1ccc2c(c1)OCO2)c1ccccc1</smiles><smiles>COc1c(/C=C/C(=O)Cc2ccccc2)cc2c(c1OC)OCO2</smiles><smiles>CCCOCCOCCOCc1cc2c(cc1CCC)OCCO2</smiles>

XXXIII

material and to the standard synergist, PBO. These findings in comparison with earlier work $^{4)}$ indicate that $\alpha, \beta$-unsaturated aliphatic compounds are superior when compared with the corresponding aromatic compounds. Comparison of the activity of (XXXI) and (XXXII) with rest of the test chemicals shows that a shift in the position of the carbonyl moiety does not seem to significantly influence the synergistic activity.

Thus the results show that the synergistic effect of this type of pyrethrum synergist depends only partly on their lipophilicity, and the introduction of polar groups like nitro and chloro as in XXII, XXIII, XXVIII and XXIX reduces the effect drastically. None of the compounds now reported are a better synergist than furapiole, although some of them are comparable to PBO.

\section{EXPERIMENTAL}

IR spectra were recorded on a Perkin Elmer-457 spectrophotometer. NMR spectra were recorded on a Varian A-60 spectrometer using TMS as the internal reference; chemical shifts are given in $\delta$ values. Dihydrodillapiole and furapiole were synthesized as reported earlier., ${ }^{4,5}$

General procedure for acylation of dihydrodillapiole (I) 
and furapiole (II). To a well stirred solution of the appropriate acid chloride $(0.15 \mathrm{~mol})$ and fused zinc chloride $(0.1 \mathrm{~mol})$ in dry methylene chloride $(150 \mathrm{ml})$, was added dropwise dihydrodillapiole or furapiole $(0.1 \mathrm{~mol})$. The reaction mixture was stirred for $3 \sim 4 \mathrm{hr}$ in an ice-bath, poured into cold water and extracted with methylene chloride. The extract was then washed free of acid and dried over $\mathrm{Na}_{2} \mathrm{SO}_{4}$. The acyl derivatives of dihydrodillapiole (III $\sim$ XII) were obtained as colourless liquids after distillation under reduced pressure at an oil bath temperature of $200^{\circ} \mathrm{C}$ (yield $\sim 70 \%$ ). The acyl derivatives of furapiole (XIII $\sim$ XIX) were obtained as colourless solids on crystallization from suitable solvents. All the acyl derivatives gave characteristic carbonyl peaks at $1660 \sim 1670 \mathrm{~cm}^{-1}$ in their IR spectra. The following acyl derivatives of dihydrodillapiole and furapiole were synthesized by this method.

Acetyl dihydrodillapiole (III). Anal. Found: C, 63.3; $\mathrm{H}$, 7.3. Calcd. for $\mathrm{C}_{14} \mathrm{H}_{18} \mathrm{O}_{5}: \mathrm{C}, 63.2 ; \mathrm{H}, 6.8 \%$. PMR $\left(\mathrm{CDCl}_{3}\right)$ $0.91\left(3 \mathrm{H}, \mathrm{t}, J=6.5 \mathrm{~Hz}\right.$. $\left.-\mathrm{CH}_{2} \mathrm{CH}_{2} \mathrm{CH}_{3}\right), 1.40(2 \mathrm{H}, \mathrm{m}$, $\left.-\mathrm{CH}_{2} \mathrm{CH}_{2} \mathrm{CH}_{3}\right), 2.4\left(3 \mathrm{H}, \mathrm{s},-\mathrm{COCH}_{3}\right), 2.6(2 \mathrm{H}, \mathrm{t}, J=$ $\left.6.5 \mathrm{~Hz}, \mathrm{ArCH}_{2}\right), 3.66\left(3 \mathrm{H}, \mathrm{s}, \mathrm{OCH}_{3}\right), 3.97\left(3 \mathrm{H}, \mathrm{s}, \mathrm{OCH}_{3}\right)$, $5.88\left(2 \mathrm{H}, \mathrm{s},-\mathrm{OCH}_{2} \mathrm{O}-\right)$.

Propionyl dihydrodillapiole (IV). Anal. Found: C, 63.9; $\mathrm{H}, 6.9$. Calcd. for $\mathrm{C}_{15} \mathrm{H}_{20} \mathrm{O}_{5}: \mathrm{C}, 64.3 ; \mathrm{H}, 7.1 \%$. PMR $\left(\mathrm{CCl}_{4}\right) 0.92\left(6 \mathrm{H}, \mathrm{m}, 2 \mathrm{xCH}_{3}\right), 1.2 \sim 1.6(2 \mathrm{H}$, br $\mathrm{m}$, $\left.\mathrm{CH}_{2} \mathrm{CH}_{3}\right), 2.4 \sim 2.9\left(4 \mathrm{H}, \mathrm{m}, \mathrm{ArC}_{2}-\right.$ and $\left.-\mathrm{COCH}_{2} \mathrm{CH}_{3}\right)$, $3.67\left(3 \mathrm{H}, \mathrm{s}, \mathrm{OCH}_{3}\right), 3.98\left(3 \mathrm{H}, \mathrm{s}, \mathrm{OCH}_{3}\right), 5.86(2 \mathrm{H}, \mathrm{s}$, $\left.-\mathrm{OCH}_{2} \mathrm{O}_{-}\right)$.

Butyryl dihydrodillapiole (V). Anal. Found: C, 65.8; H, 8.0. Calcd. for $\mathrm{C}_{16} \mathrm{H}_{22} \mathrm{O}_{5}: \mathrm{C}, 65.3 ; \mathrm{H}, 7.5 \%$. PMR $\left(\mathrm{CCl}_{4}\right)$ $0.92\left(6 \mathrm{H}, \quad \mathrm{t}, \quad J=7 \mathrm{~Hz}, 2 \mathrm{X}-\mathrm{CH}_{3}\right), 1.2 \sim 1.8(4 \mathrm{H}, \mathrm{m}$, $\left.2 \times \underline{\mathrm{H}}_{2} \mathrm{CH}_{3}\right), 2.3 \sim 2.83\left(4 \mathrm{H}, \mathrm{m}, \mathrm{ArCH}_{2}-\right.$ and $\left.-\mathrm{COCH}_{2}-\right)$, $3.67\left(3 \mathrm{H}, \mathrm{s}, \mathrm{OCH}_{3}\right), 3.93\left(3 \mathrm{H}, \mathrm{s}, \mathrm{OCH}_{3}\right) ; 5.85(2 \mathrm{H}, \mathrm{s}$, $-\mathrm{OC}_{2} \mathrm{O}-$ ).

Valeryl dihydrodillapiole (VI). Anal. Found: C, 65.9; $\mathrm{H}$, 8.1. Calcd. for $\mathrm{C}_{17} \mathrm{H}_{24} \mathrm{O}_{5}$ : C, 66.2; $\mathrm{H}, 7.8 \%$. PMR $\left(\mathrm{CCl}_{4}\right)$ : $0.92\left(6 \mathrm{H}, \mathrm{m}, 2 \mathrm{x}-\mathrm{CH}_{3}\right), 1.15 \sim 1.75(6 \mathrm{H}$, br m, methylenic protons); $2.15 \sim 2.85\left(4 \mathrm{H}, \mathrm{m}, \mathrm{ArCH}_{2}\right.$ and $\left.-\mathrm{COCH}_{2}-\right), 3.68$ $\left(3 \mathrm{H}, \mathrm{s}, \mathrm{OCH}_{3}\right), 3.95\left(3 \mathrm{H}, \mathrm{s}, \mathrm{OCH}_{3}\right), 5.85(2 \mathrm{H}, \mathrm{s}$, $-\mathrm{OC}_{2} \mathrm{O}-$ ).

Caproyl dihydrodillapiole (VII). Anal. Found: C, 66.8; $\mathrm{H}$, 8.5. Calcd. for $\mathrm{C}_{18} \mathrm{H}_{26} \mathrm{O}_{5}$ : C, 67.1; $\mathrm{H}, 8.1 \%$. $\operatorname{PMR}\left(\mathrm{CCl}_{4}\right): 0.9\left(6 \mathrm{H}, \mathrm{m}, 2 \mathrm{xCH}_{3}\right), 1.2 \sim 1.8(8 \mathrm{H}$, br m, methylenic protons), $2.4 \sim 2.95\left(4 \mathrm{H}, \mathrm{m}, \mathrm{ArCH}_{2}-\right.$ and $\left.\mathrm{COC}_{\mathrm{H}_{2}}-\right), 3.67\left(3 \mathrm{H}, \mathrm{s}, \mathrm{OC}_{3}\right), 3.95\left(3 \mathrm{H}, \mathrm{s}, \mathrm{OCH}_{3}\right)$, $5.94\left(2 \mathrm{H}, \mathrm{s},-\mathrm{OCH}_{2} \mathrm{O}-\right)$.

Heptanoyl dihydrodillapiole (VIII). Anal. Found: C, 68.2; $\mathrm{H}, 8.7$. Calcd. for $\mathrm{C}_{19} \mathrm{H}_{28} \mathrm{O}_{5}$ : C, 67.9; $\mathrm{H}, 8.3 \%$. PMR $\left(\mathrm{CCl}_{4}\right) 0.92\left(6 \mathrm{H}, \mathrm{m}, 2 \mathrm{xCH}_{3}\right), 1.1 \sim 1.8(10 \mathrm{H}$, br m, methylenic protons), $2.46 \sim 2.83\left(4 \mathrm{H}, \mathrm{m}, \mathrm{ArC}_{2}\right.$ and
$\left.-\mathrm{COCH}_{2}-\right), 3.68\left(3 \mathrm{H}, \mathrm{s}, \mathrm{OCH}_{3}\right), 3.95\left(3 \mathrm{H}, \mathrm{s}, \mathrm{OCH}_{3}\right)$, $5.81\left(2 \mathrm{H}, \mathrm{s},-\mathrm{OCH}_{2} \mathrm{O}-\right)$.

Octanoyl dihydrodillapiole (IX). Anal. Found: C, 69.0; $\mathrm{H}$, 9.0. Calcd. for $\mathrm{C}_{20} \mathrm{H}_{30} \mathrm{O}_{5}: \mathrm{C}, 68.6 ; \mathrm{H}, 8.6 \%$. PMR $\left(\mathrm{CCl}_{4}\right) ; 0.90\left(6 \mathrm{H}, \mathrm{m}, 2 \mathrm{xCH}_{3}\right), 1.05 \sim 1.8(12 \mathrm{H}$, br $\mathrm{m}$, methylenic protons) $2.2 \sim 2.8\left(4 \mathrm{H}, \mathrm{m}, \mathrm{ArCH}_{2}\right.$ and $-\mathrm{COC}_{2}-$ ) $3.67\left(3 \mathrm{H}, \mathrm{s}, \mathrm{OCH}_{3}\right), 3.95\left(3 \mathrm{H}, \mathrm{s}, \mathrm{OC}_{3}\right), 5.85$ $\left(2 \mathrm{H}, \mathrm{s},-\mathrm{OCH}_{2} \mathrm{O}-\right)$.

Pelargonyl dihydrodillapiole (X). Anal. Found: C, 69.1; $\mathrm{H}$, 9.2. Calcd. for $\mathrm{C}_{21} \mathrm{H}_{32} \mathrm{O}_{5}: \mathrm{C}, 69.2 ; \mathrm{H}, 8.8 \%$. PMR $\left(\mathrm{CCl}_{4}\right): 0.91\left(\mathrm{~m}, 6 \mathrm{H}, 2 \mathrm{xCH}_{3}\right), 1.2 \sim 1.8(14 \mathrm{H}$, br $\mathrm{m}$, methylenic protons), $2.3 \sim 2.8\left(4 \mathrm{H}, \mathrm{m}, \mathrm{ArCH}_{2}\right.$ and $\left.-\mathrm{COC}_{\mathrm{H}_{2}}-\right), 3.67\left(3 \mathrm{H}, \mathrm{s}, \mathrm{OC}_{3}\right), 3.95\left(3 \mathrm{H}, \mathrm{s}, \mathrm{OC}_{3}\right)$, $5.85\left(2 \mathrm{H}, \mathrm{s},-\mathrm{OCH}_{2} \mathrm{O}-\right)$.

Decanoyl dihydrodillapiole (XI). Anal. Found: C, 70.0; $\mathrm{H}$, 9.4. Calcd. for $\mathrm{C}_{22} \mathrm{H}_{34} \mathrm{O}_{5}: \mathrm{C}, 69.9 ; \mathrm{H}, 9.1 \%$. PMR $\left(\mathrm{CCl}_{4}\right): 0.9\left(6 \mathrm{H}, \mathrm{m}, 2 \mathrm{xCH}_{3}\right), 1.15 \sim 1.75(16 \mathrm{H}$, br $\mathrm{m}$, methylenic protons), $2.38 \sim 2.88\left(4 \mathrm{H}, \mathrm{m}, \mathrm{ArCH}_{2}\right.$ and $\left.-\mathrm{COCH}_{2}-\right)_{6} 3.78\left(3 \mathrm{H}, \mathrm{s}, \mathrm{OCH}_{3}\right), 3.96\left(3 \mathrm{H}, \mathrm{s}, \mathrm{OC}_{3}\right), 5.86$ $\left(2 \mathrm{H}, \mathrm{s},-\mathrm{OC}_{2} \mathrm{O}-\right)$.

Chloroacetyl dihydrodillapiole (XII). Anal. Found: C, 56.2; $\mathrm{H}, 6.1$. Calcd. for $\mathrm{C}_{14} \mathrm{H}_{17} \mathrm{O}_{5} \mathrm{Cl}: \mathrm{C}, 55.9 ; \mathrm{H}, 5.7 \%$. $\operatorname{PMR}\left(\mathrm{CDCl}_{3}\right): 0.82\left(3 \mathrm{H}, \mathrm{t}, J=7 \mathrm{~Hz},-\mathrm{CH}_{2} \mathrm{CH}_{2} \mathrm{CH}_{3}\right), 1.15$ $\left(2 \mathrm{H}, \mathrm{m}, \mathrm{CH}_{2} \mathrm{CH}_{3}\right), 2.51\left(2 \mathrm{H}, \mathrm{t}, J=7 \mathrm{~Hz}, \mathrm{ArCH}_{2}-\right), 3.73$ $\left(3 \mathrm{H}, \mathrm{s}, \mathrm{OCH}_{3}\right), 3.82\left(2 \mathrm{H}, \mathrm{s},-\mathrm{COC}_{2} \mathrm{Cl}\right), 3.96(3 \mathrm{H}, \mathrm{s}$, $\left.\mathrm{OC}_{3}\right), 5.83\left(2 \mathrm{H}, \mathrm{s},-\mathrm{OCH}_{2} \mathrm{O}-\right)$.

Acetylfurapiole (XIII). Crystallized from pet. ether as colourless needles, mp $109^{\circ} \mathrm{C}$. Anal. Found: C, 62.2; H, 6.1, Calcd. for $\mathrm{C}_{13} \mathrm{H}_{14} \mathrm{O}_{5}: \mathrm{C}, 62.4 ; \mathrm{H}, 5.6 \%$. PMR $\left(\mathrm{CDCl}_{3}\right): 1.45\left(\mathrm{~d}, 3 \mathrm{H}, J=6.5 \mathrm{~Hz},-\mathrm{CH}-\mathrm{CH}_{3}\right), 2.5(\mathrm{~s}, 3 \mathrm{H}$, $\left.-\mathrm{COCH}_{3}\right), 3.65\left(\mathrm{~m}, 2 \mathrm{H}, \mathrm{Ar}-\mathrm{CH}_{2}-\right), 4.09\left(\mathrm{~s}, 3 \mathrm{H}, \mathrm{OCH}_{3}\right)$, $4.9\left(\mathrm{~m}, 1 \mathrm{H}, \mathrm{O}-\mathrm{CHCH}_{3}\right), 5.95\left(\mathrm{~s}, 2 \mathrm{H}, \mathrm{OCH}_{2} \mathrm{O}\right)$.

Propionyl furapiole (XIV). Crystallized from pet. ether as colourless needles, mp $95^{\circ} \mathrm{C}$. Anal. Found: C, 64.1; H, 6.5. Calcd. for $\mathrm{C}_{14} \mathrm{H}_{16} \mathrm{O}_{5}: \mathrm{C}, 63.6 ; \mathrm{H}, 6.0 \%$. PMR $\left(\mathrm{CDCl}_{3}\right): 1.2\left(\mathrm{t}, 3 \mathrm{H}, J=7 \mathrm{~Hz}, \mathrm{COCH}_{2} \mathrm{CH}_{3}\right), 1.5(\mathrm{~d}, 3 \mathrm{H}$, $\left.J=6.5 \mathrm{~Hz}, \mathrm{CHCH}_{3}\right), 2.9\left(\mathrm{t}, 2 \mathrm{H}, J=7 \mathrm{~Hz}, \mathrm{COCH}_{2} \mathrm{CH}_{3}\right)$, $3.65\left(\mathrm{~m}, 2 \mathrm{H}, \mathrm{ArCH}_{2}\right), 4.12\left(\mathrm{~s}, 3 \mathrm{H}, \mathrm{OCH}_{3}\right) ; 4.9(\mathrm{~m}, 1 \mathrm{H}$, $\left.\mathrm{OCH}_{\mathrm{HCH}}\right) 5.94\left(\mathrm{~s}, 2 \mathrm{H}, \mathrm{OCH}_{2} \mathrm{O}\right)$.

Butyryl furapiole (XV). Crystallized from pet. ether as colourless needles, $\mathrm{mp} 87^{\circ} \mathrm{C}$. Anal. Found: C, 64.7; H, 6.4. Calcd. for $\mathrm{C}_{15} \mathrm{H}_{18} \mathrm{O}_{5}$ : C, 64.7; H, 6.5\%. PMR $\left(\mathrm{CDCl}_{3}\right) 1.0$ $\left(\mathrm{t}, 3 \mathrm{H}, J=7 \mathrm{~Hz}, \mathrm{CH}_{2} \mathrm{CH}_{3}\right) ; 1.5\left(\mathrm{~m}, 5 \mathrm{H}, \mathrm{C}_{2}-\mathrm{CH}_{3}\right.$ and $\left.\mathrm{CH}-\mathrm{CH}_{3}\right), 2.8\left(\mathrm{t}, 2 \mathrm{H}, J=7 \mathrm{~Hz}, \mathrm{COCH}_{2} \mathrm{CH}_{2}-\right) ; 3.6(\mathrm{~m}, 2 \mathrm{H}$, $\left.\mathrm{ArC}_{\underline{H}}\right), 4.1\left(\mathrm{~s}, 3 \mathrm{H},-\mathrm{OC}_{3}\right), 4.95\left(\mathrm{~m}, 1 \mathrm{H}-\mathrm{C}_{\underline{H C H}}\right), 6.0(\mathrm{~s}$, $2 \mathrm{H},-\mathrm{OCH}_{2} \mathrm{O}-$ ).

Heptanoyl furapiole (XVI). Crystallized from pet. ether as colourless plates mp $58^{\circ} \mathrm{C}$. Anal. Found: C, 67.1; H, 7.9. Calcd. for $\mathrm{C}_{18} \mathrm{H}_{24} \mathrm{O}_{5}: \mathrm{C}, 67.5 ; \mathrm{H}, 7.5 \%$. PMR $\left(\mathrm{CDCl}_{3}\right) 0.9$ 
(t, $\left.3 \mathrm{H}, \quad J=7 \mathrm{~Hz}, \quad-\mathrm{C}_{2} \mathrm{CH}_{3}\right) ; 1.15 \sim 1.9 \quad(\mathrm{~m}, \quad 11 \mathrm{H}$, $\left(\mathrm{CH}_{2}\right)_{4}-\mathrm{CH}_{3}$ and $\left.-\mathrm{CHCH}_{3}\right), 2.8(\mathrm{t}, \quad 2 \mathrm{H}, \quad J=7 \mathrm{~Hz}$,

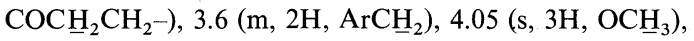
$4.9\left(\mathrm{~m}, 1 \mathrm{H},-\mathrm{OCHCH}_{3}\right), 5.95\left(\mathrm{~s}, 2 \mathrm{H},-\mathrm{OCH}_{2} \mathrm{O}-\right)$.

Octanoyl furapiole (XVII). Crystallized from pet. ether as colourless plates, $\mathrm{mp} 55^{\circ} \mathrm{C}$. Anal. Found: C, 68.0; $\mathrm{H}$, 7.8. Calcd. for $\mathrm{C}_{19} \mathrm{H}_{26} \mathrm{O}_{5}: \mathrm{C}, 68.3 ; \mathrm{H}, 7.8 \%$. PMR $\left(\mathrm{CDCl}_{3}\right)$ $0.9\left(\mathrm{t}, 3 \mathrm{H}, J=7 \mathrm{~Hz}, \mathrm{CH}_{2} \mathrm{CH}_{3}\right), 1.20 \sim 1.8(\mathrm{~m}, 13 \mathrm{H}$, $-\left(\mathrm{CH}_{2}\right)_{5} \mathrm{CH}_{3}$ and $\left.-\mathrm{CHCH}_{3}\right), 2.86(\mathrm{t}, 2 \mathrm{H}, \quad J=7 \mathrm{~Hz}$,

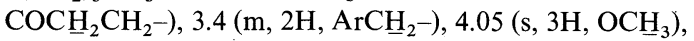
$4.9\left(\mathrm{~m}, 1 \mathrm{H}, \mathrm{OC}^{\mathrm{HCH}} \mathrm{H}_{3}\right), 5.98\left(\mathrm{~s}, 2 \mathrm{H},-\mathrm{OCH}_{2} \mathrm{O}_{-}\right)$.

Decanoyl furapiole (XVIII). Crystallized from pet. ether as colourless powder, mp $53^{\circ} \mathrm{C}$. Anal. Found: C, 69.7; $\mathrm{H}$, 8.5. Calcd. for $\mathrm{C}_{21} \mathrm{H}_{30} \mathrm{O}_{5}: \mathrm{C}, 69.6 ; \mathrm{H}, 8.3 \%$. PMR $\left(\mathrm{CDCl}_{3}\right)$ $0.85\left(\mathrm{t}, 3 \mathrm{H}, J=7 \mathrm{~Hz}, \mathrm{CH}_{2} \mathrm{CH}_{3}\right), 1.15 \sim 1.6(\mathrm{~m}, 17 \mathrm{H}$, $-\mathrm{CH}_{2}\left(\mathrm{CH}_{2}\right)_{7} \mathrm{CH}_{3}$ and $\left.-\mathrm{CHCH}_{3}\right), 2.9(\mathrm{t}, 2 \mathrm{H}, J=7 \mathrm{~Hz}$, $\left.-\mathrm{COC}_{2} \mathrm{CH}_{2}\right), 3.4\left(\mathrm{~m}, 2 \mathrm{H}, \mathrm{ArCH}_{2}-\right) ; 4.09\left(\mathrm{~s}, 3 \mathrm{H}, \mathrm{OCH}_{3}\right)$; $5.0\left(\mathrm{~m}, 1 \mathrm{H},-\mathrm{OC}^{\mathrm{HCH}} \mathrm{H}_{3}\right) ; 5.95\left(\mathrm{~s}, 2 \mathrm{H},-\mathrm{OC}_{2}-\mathrm{O}-\right)$.

Chloroacetylfurapiole (XIX). Crystallized from methanol as colourless needles, mp $147 \sim 149^{\circ} \mathrm{C}$. Anal. Found: $\mathrm{C}, 55.1 ; \mathrm{H}, 5.0$. Calcd. for $\mathrm{C}_{13} \mathrm{H}_{13} \mathrm{O}_{5} \mathrm{Cl}: \mathrm{C}, 54.8 ; \mathrm{H}$, $4.6 \%$. PMR $\left(\mathrm{CDCl}_{3}\right) 1.38\left(\mathrm{~d}, 3 \mathrm{H}, J=7 \mathrm{~Hz},-\mathrm{OCHCH}_{3}\right)$, $2.3 \sim 3.2$ (br m, $2 \mathrm{H}, \mathrm{ArCH}_{2}$ ), 3.7 (s, $2 \mathrm{H}, \mathrm{COCH}_{2} \mathrm{Cl}$ ); 3.94 $\left(\mathrm{s}, 3 \mathrm{H}, \mathrm{OCH}_{3}\right), 4.8\left(\mathrm{~m}, 1 \mathrm{H}, \mathrm{OCH}^{\mathrm{H}} \mathrm{CH}_{3}\right), 5.8(\mathrm{~s}, 2 \mathrm{H}$, $-\mathrm{OCH}_{2} \mathrm{O}-$ ).

General procedure for the synthesis of $\alpha, \beta$-unsaturated ketones from Acetyl dihydrodillapiole and Acetyl furapiole ( $\mathbf{X X} \sim \mathbf{X X X}$ ). To a well-stirred mixture of acetyl dihydrodillapiole or acetyl furapiole $(0.1 \mathrm{~mol})$, aqueous $\mathrm{NaOH}$ $(20 \%, 10 \mathrm{ml})$ and ethanol $(20 \mathrm{ml})$ maintained at a low temperature (ice bath) was added the appropriate aldehyde $(0.1 \mathrm{~mol})$ in ethanol $(10 \mathrm{ml})$. The reaction mixture was gradually brought to room temperature and stirred until it became a semisolid viscous mass $(2 \sim 3 \mathrm{hr})$. Water $(100 \mathrm{ml})$ was added. The precipitated product was filtered and washed with cold water until free from alkali. It was finally crystallized from rectified spirit (yield $60 \sim 70 \%$ ). All these compounds gave a characteristic peak at $1665 \sim 1670 \mathrm{~cm}^{-1}$ for a carbonyl group, in their IR spectra. 3,4-Methylenedioxy cinnamoyl benzene (XXXI) was similarly prepared by condensation of piperonal and acetophenone. 2,3-Dimethoxy-4,5-methylenedioxy cinnamoyl benzene (XXXII) was prepared from dillapiole aldehyde and acetophenone in a similar manner.

Cinnamoyl dihydrodillapiole (XX). Crystallized from methanol as light yellow needles mp $75^{\circ} \mathrm{C}$. Anal. Found: C, 70.9; H, 5.5. Calcd. for $\mathrm{C}_{21} \mathrm{H}_{22} \mathrm{O}_{5}: \mathrm{C}, 71.2, \mathrm{H}, 6.20 \%$. $\operatorname{PMR}\left(\mathrm{CDCl}_{3}\right) 0.95\left(\mathrm{t}, 3 \mathrm{H}, J=7 \mathrm{~Hz},-\mathrm{CH}_{2} \mathrm{CH}_{3}\right) ; 1.5(\mathrm{~m}$, $\left.2 \mathrm{H},-\mathrm{CH}_{2} \mathrm{CH}_{3}\right) ; 2.65\left(\mathrm{t}, 2 \mathrm{H}, J=7 \mathrm{~Hz}, \mathrm{ArCl}_{2}-\right) ; 3.78(\mathrm{~s}$,

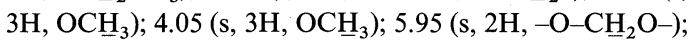
$7.1 \sim 7.7\left(\mathrm{~m}, 7 \mathrm{H}, \mathrm{C}_{6} \underline{\mathrm{H}}_{5} \mathrm{C} \underline{\mathrm{H}}=\mathrm{C} \underline{\mathrm{H}}-\right)$.
3,4-Methylenedioxy cinnamoyl dihydrodillapiole (XXI). Crystallized from methanol as yellow needles, mp $95^{\circ} \mathrm{C}$. Anal. Found: C, 66.9; $\mathrm{H}, 6.0$. Calcd. for $\mathrm{C}_{22} \mathrm{H}_{22} \mathrm{O}_{7}$ : C, $66.3 ; \mathrm{H}, 5.5 \%$. PMR $\left(\mathrm{CDCl}_{3}\right) .0 .95(\mathrm{t}, 3 \mathrm{H}, J=7 \mathrm{~Hz}$, $\left.\mathrm{CH}_{2} \mathrm{CH}_{3}\right) ; 1.5\left(\mathrm{~m}, 2 \mathrm{H}, \mathrm{CH}_{2} \mathrm{CH}_{3}\right) ; 2.65(\mathrm{t}, 2 \mathrm{H}, J=7 \mathrm{~Hz}$, $\left.\mathrm{ArC} \underline{H}_{2}-\right), 3.8\left(\mathrm{~s}, 3 \mathrm{H}, 2 \mathrm{xOCH}_{3}\right), 4.06\left(\mathrm{~s}, 3 \mathrm{H}, \mathrm{OCH}_{3}\right)$ $5.86 \& 5.95\left(\mathrm{~s}, 4 \mathrm{H}, 2 \mathrm{xOCH}_{2} \mathrm{O}-\right), 6.6 \sim 7.6(\mathrm{~m}, 5 \mathrm{H}$, $\left.\mathrm{C}_{6} \underline{\mathrm{H}}_{3} \mathrm{C} \underline{\mathrm{H}}=\mathrm{C} \underline{\mathrm{HCO}}\right)$.

4-Nitrocinnamoyl dihydrodillapiole (XXII). Crystallized from $\mathrm{MeOH}$ as bright yellow needles, mp $149 \sim 150^{\circ} \mathrm{C}$. Anal. Found: C, 63.4; H, 5.8. Calcd. for $\mathrm{C}_{21} \mathrm{H}_{21} \mathrm{NO}_{7}$ : C, 63.2; H, 5.3\%. PMR $\left(\mathrm{CDCl}_{3}\right) 1.0(\mathrm{t}, 3 \mathrm{H}, J=7 \mathrm{~Hz}$, $\left.-\mathrm{CH}_{2} \mathrm{CH}_{3}\right), 1.6\left(\mathrm{~m}, 2 \mathrm{H}, \mathrm{CH}_{2} \mathrm{CH}_{3}\right), 2.8(\mathrm{t}, 2 \mathrm{H}, J=7 \mathrm{~Hz}$, $\left.\mathrm{ArCH}_{2}\right) ; 3.8\left(\mathrm{~s}, 3 \mathrm{H}, \mathrm{OCH}_{3}\right), 4.14\left(\mathrm{~s}, 3 \mathrm{H}, \mathrm{OCH}_{3}\right), 5.98(\mathrm{~s}$, $\left.2 \mathrm{H},-\mathrm{OCH}_{2} \mathrm{O}-\right), 7.2 \sim 7.9\left(\mathrm{~m}, 5 \mathrm{H}, \mathrm{C}_{6} \underline{\mathrm{H}}_{4}-\mathrm{CH}=\mathrm{CHCO}-\right)$; $8.2(\mathrm{~d}, 1 \mathrm{H}, J=9 \mathrm{~Hz}, \mathrm{Ar}-\mathrm{CH}=\mathrm{CH}-$ ).

4-Chloro cinnamoyl dihydrodillapiole (XXIII). Crystallized from methanol as yellow coloured needles, $\mathrm{mp}$ $102 \sim 103^{\circ} \mathrm{C}$. Anal. Found: C, 65.0; H, 6.0; Calcd. for $\mathrm{C}_{21} \mathrm{H}_{21} \mathrm{O}_{5} \mathrm{Cl}: \mathrm{C}, 64.9 ; \mathrm{H}, 5.4 \%$. PMR $\left(\mathrm{CDCl}_{3}\right), 1.0(\mathrm{t}, 3 \mathrm{H}$, $\left.J=7 \mathrm{~Hz}, \mathrm{CH}_{2} \mathrm{CH}_{3}\right) ; 1.2\left(\mathrm{~m}, 2 \mathrm{H}, \mathrm{CH}_{2} \mathrm{CH}_{3}\right), 2.7(\mathrm{t}, 2 \mathrm{H}, J=$ $\left.7 \mathrm{~Hz}, \mathrm{ArCH}_{2}-\right), 3.8\left(\mathrm{~s}, 3 \mathrm{H}, \mathrm{OCH}_{3}\right), 4.1\left(\mathrm{~s}, 3 \mathrm{H}, \mathrm{OCH}_{3}\right), 5.95$ (s, $\left.2 \mathrm{H},-\mathrm{OCH}_{2} \mathrm{O}-\right), 7.2 \sim 7.8\left(\mathrm{~m}, 6 \mathrm{H}, \mathrm{C}_{6} \underline{\mathrm{H}}_{4} \mathrm{C} \underline{\mathrm{H}}=\mathrm{C} \underline{\mathrm{H}}-\right)$.

3-(2-Furylpropenoyl) dihydrodillapiole (XXIV). Light yellow coloured viscous liquid. Anal. Found: C, 65.9; H, 6.2. Calcd. for $\mathrm{C}_{19} \mathrm{H}_{20} \mathrm{O}_{6}: \mathrm{C}, 66.3 ; \mathrm{H}, 5.8 \%$. PMR $\left(\mathrm{CDCl}_{3}\right), 0.9\left(\mathrm{t}, 3 \mathrm{H}, J=7 \mathrm{~Hz}, \mathrm{CH}_{2} \mathrm{CH}_{3}\right), 1.5(\mathrm{~m}, 2 \mathrm{H}$, $\left.\mathrm{C}_{2} \mathrm{CH}_{3}\right), 2.65\left(\mathrm{t}, 2 \mathrm{H}, J=7 \mathrm{~Hz}, \mathrm{ArC}_{2}-\right), 3.78(\mathrm{~s}, 3 \mathrm{H}$, $\left.\mathrm{OCH}_{3}\right), 4.06\left(\mathrm{~s}, 3 \mathrm{H}, \mathrm{OCH}_{3}\right), 5.91\left(\mathrm{~s}, 2 \mathrm{H},-\mathrm{OCH}_{2} \mathrm{O}_{-}\right)$, $6.5 \sim 7.52\left(\mathrm{~m}, 5 \mathrm{H}, \mathrm{C}_{4} \underline{\mathrm{H}}_{3} \mathrm{O}-\mathrm{C} \underline{\mathrm{H}}=\mathrm{C} \underline{\mathrm{HCO}}\right)$.

2-Nonenoyl dihydrodillapiole (XXV). Colourless viscous liquid. Anal. Found: C, 70.0; H, 8.7. Calcd. for $\mathrm{C}_{21} \mathrm{H}_{30} \mathrm{O}_{5}$ : $\mathrm{C}, 69.6 ; \mathrm{H}, 8.3 \%$. PMR $\left(\mathrm{CDCl}_{3}\right) 0.9(\mathrm{t}, 6 \mathrm{H}, J=7 \mathrm{~Hz}$, side chain methyls), $1.1 \sim 2.0$ (br m, $12 \mathrm{H}, \mathrm{C}_{2} \mathrm{CH}_{3}$ and $\left.-\left(\mathrm{C}_{2}\right)_{5}-\mathrm{CH}_{3}\right), 2.5\left(\mathrm{t}, 2 \mathrm{H}, J=7 \mathrm{~Hz}, \mathrm{ArCl}_{2}-\right), 3.3 \sim 3.78$ $(\mathrm{m}, 1 \mathrm{H}, \mathrm{COCH}=\mathrm{CH}-), 3.8\left(\mathrm{~s}, 3 \mathrm{H}, \mathrm{OCH}_{3}\right), 4.06(\mathrm{~s}, 3 \mathrm{H}$, $\left.\mathrm{OCH}_{3}\right), 5.85(\mathrm{~d}, 1 \mathrm{H}, J=10 \mathrm{~Hz},-\mathrm{COC} \underline{\mathrm{H}}=\mathrm{CH}-), 5.95$ (s, $2 \mathrm{H},-\mathrm{OCH}_{2} \mathrm{O}-$ ).

Cinnamoyl furapiole (XXVI). Crystallized from $\mathrm{MeOH}$ as yellow needles mp $124^{\circ} \mathrm{C}$. Anal. Found: C, 70.9; H, 5.5. Calcd. for $\mathrm{C}_{20} \mathrm{H}_{18} \mathrm{O}_{5} ; \mathrm{C}, 71.0 ; \mathrm{H}, 5.3 \%$. PMR $\left(\mathrm{CDCl}_{3}\right) 1.4$ (d, $\left.3 \mathrm{H}, J=6.5 \mathrm{~Hz},-\mathrm{CHCH}_{3}\right), 3.4\left(\mathrm{~m}, 2 \mathrm{H}, \mathrm{ArCH}_{2}-\right), 4.05$ (s, $\left.3 \mathrm{H}, \mathrm{OCH}_{3}\right) 4.9\left(\mathrm{~m}, 1 \mathrm{H},-\mathrm{CHCH}_{3}\right), 5.95(\mathrm{~s}, 2 \mathrm{H}$, $\left.-\mathrm{OC}_{2} \mathrm{O}\right), 7.1 \sim 7.8\left(\mathrm{~m}, 7 \mathrm{H}, \mathrm{C}_{6} \underline{\mathrm{H}}_{5}-\mathrm{C} \underline{\mathrm{H}}=\mathrm{C} \underline{\mathrm{H}}-\right)$.

3,4-Methylenedioxycinnamioyl furapiole (XXVII). Crystallized from $\mathrm{MeOH}$ as bright yellow needles, $\mathrm{mp}$ $178^{\circ}$ C. Anal. Found: C, 65.8; H, 5.0. Calcd. for $\mathrm{C}_{21} \mathrm{H}_{18} \mathrm{O}_{7}$ : C, 66.0; H, 4.7\%. PMR $\left(\mathrm{CDCl}_{3}\right) 1.5(\mathrm{~d}, 3 \mathrm{H}, J=6.5 \mathrm{~Hz}$, $\left.\mathrm{CHCH}_{3}\right) 3.45\left(\mathrm{~m}, 2 \mathrm{H}, \mathrm{ArCH}_{2}\right), 4.1\left(\mathrm{~s}, 3 \mathrm{H}, \mathrm{OC}_{3}\right), 5.0(\mathrm{~m}$, $\left.1 \mathrm{H},-\mathrm{CHCH}_{3}\right), 5.98\left(\mathrm{~s}, 2 \mathrm{H},-\mathrm{OCH}_{2} \mathrm{O}\right), 6.73(\mathrm{~d}, 1 \mathrm{H}, J=$ $9 \mathrm{~Hz}, \mathrm{Ar}-\mathrm{CH}=\mathrm{CHCO}) ; 7.05$ (m, 3H, aromatic protons), 
$7.5(\mathrm{~d}, 1 \mathrm{H}, J=9 \mathrm{~Hz}, \mathrm{Ar}-\mathrm{C} \underline{\mathrm{H}}=\mathrm{CH}-)$.

4-Nitrocinnamoyl furapiole (XXVIII). Crystallized from $\mathrm{MeOH}$ as orange coloured needles, mp $233 \sim 235^{\circ} \mathrm{C}$. Anal. Found: $\mathrm{C}, 63.0 ; \mathrm{H}, 4.0$. Calcd. for $\mathrm{C}_{20} \mathrm{H}_{17} \mathrm{NO}_{7}: \mathrm{C}, 62.7 ; \mathrm{H}$, $4.4 \%$. PMR $\left(\mathrm{CDCl}_{3}\right) 1.45\left(\mathrm{~d}, 3 \mathrm{H}, J=6.5 \mathrm{~Hz},-\mathrm{CHCH}_{3}\right)$, $3.3\left(\mathrm{~m}, 2 \mathrm{H}, \mathrm{ArC}_{2}-\right) ; 4.1\left(\mathrm{~s}, 3 \mathrm{H}, \mathrm{OCH}_{3}\right), 4.9(\mathrm{~m}, 1 \mathrm{H}$, $\left.-\mathrm{C}^{\mathrm{HCH}} \mathrm{CH}_{3}\right) ; 6.05\left(\mathrm{~s}, 2 \mathrm{H},-\mathrm{OC}_{2} \mathrm{O}\right), 7.7\left(\mathrm{~m}, 5 \mathrm{H}, \mathrm{C}_{6} \underline{\mathrm{H}}_{4} \mathrm{CH}=\right.$ C $\underline{\mathrm{H}}-\mathrm{CO}-), 8.25(\mathrm{~d}, 1 \mathrm{H}, J=10 \mathrm{~Hz}, \mathrm{ArCH}=\mathrm{CH}-$ ).

4-Chlorocinnamoyl furapiole (XXIX). Crystallized from $\mathrm{MeOH}$ as bright yellow plates, mp $184 \sim 185^{\circ} \mathrm{C}$. Anal. Found: $\mathrm{C}, 64.1 ; \mathrm{H}, 4.8$. Calcd. for $\mathrm{C}_{20} \mathrm{H}_{17} \mathrm{O}_{5} \mathrm{Cl}: \mathrm{C}, 64.4 ; \mathrm{H}$, $4.6 \%$. PMR $\left(\mathrm{CDCl}_{3}\right) 1.46\left(\mathrm{~d}, 3 \mathrm{H}, J=6.5 \mathrm{~Hz}, \mathrm{CHCH}_{3}\right), 3.4$ $\left(\mathrm{m}, 2 \mathrm{H}, \mathrm{Ar}-\mathrm{C}_{2}-\right), 4.1\left(\mathrm{~s}, 3 \mathrm{H}, \mathrm{OCH}_{3}\right), 4.9(\mathrm{~m}, 1 \mathrm{H}$, $\left.-\mathrm{CHCH}_{3}\right), 6.06\left(\mathrm{~s}, 2 \mathrm{H},-\mathrm{OCH}_{2} \mathrm{O}\right), 7.2 \sim 7.7(\mathrm{~m}, 6 \mathrm{H}$, $\left.\mathrm{ClC}_{6} \underline{\mathrm{H}}_{4} \mathrm{CH}=\mathrm{C} \underline{\mathrm{H}}-\right)$.

3-(2-Furylpropenoyl) furapiole (XXX). Crystallized from $\mathrm{MeOH}$ as light yellow plates, $\mathrm{mp} 134 \sim 135^{\circ} \mathrm{C}$. Anal. Found: $\mathrm{C}, 66.3 ; \mathrm{H}, 5.2$. Calcd. for $\mathrm{C}_{18} \mathrm{H}_{16} \mathrm{O}_{6}: \mathrm{C}, 65.9 ; \mathrm{H}$, $4.9 \%$. PMR $\left(\mathrm{CDCl}_{3}\right) 1.2\left(\mathrm{~d}, 3 \mathrm{H}, \mathrm{CHCH}_{3}\right), 3.3(\mathrm{~m}, 2 \mathrm{H}$, $\left.\operatorname{ArC} \underline{H}_{2}\right), 3.98\left(\mathrm{~s}, 3 \mathrm{H}, \mathrm{OCH}_{3}\right), 4.9\left(\mathrm{~m}, 1 \mathrm{H},-\mathrm{CHCH}_{3}\right), 5.9$ (s, $\left.2 \mathrm{H}, \quad \mathrm{OC}_{2} \mathrm{O}-\right), 6.3 \sim 7.6\left(\mathrm{~m}, 5 \mathrm{H}, \quad \mathrm{C}_{4} \underline{\mathrm{H}}_{3}-\mathrm{OC} \underline{\mathrm{H}}=\right.$ C

3,4-Methylenedioxy cinnamoyl benzene (XXXI). Crystallized from EtOH as light yellow needles, $\mathrm{mp}$ $120 \sim 121^{\circ} \mathrm{C}$. Anal. Found: C, 76.0; H, 5.2. Calcd. for $\mathrm{C}_{16} \mathrm{H}_{12} \mathrm{O}_{3}: \mathrm{C}, 76.2 ; \mathrm{H}, 4.6 \%$. PMR $\left(\mathrm{CDCl}_{3}\right) 5.87(\mathrm{~s}, 2 \mathrm{H}$, $\left.-\mathrm{OCH}_{2} \mathrm{O}-\right), 6.65 \sim 8.2(\mathrm{~m}, 10 \mathrm{H}$, Aromatic and $-\mathrm{C} \underline{\mathrm{H}}=\mathrm{C} \underline{\mathrm{H}}-$ protons).

2,3-Dimethoxy-4,5-methylenedioxy cinnamoyl benzene (XXXII). Crystallized from $\mathrm{MeOH}$ as light yellow needles, mp $75 \sim 76^{\circ} \mathrm{C}$. Anal. Found: C, 68.9; H, 5.5. Calcd. for $\mathrm{C}_{18} \mathrm{H}_{16} \mathrm{O}_{5}: \mathrm{C}, 69.2 ; \mathrm{H}, 5.1 \%$. PMR $\left(\mathrm{CDCl}_{3}\right) 3.75(\mathrm{~s}, 3 \mathrm{H}$, $\left.\mathrm{OC}_{3}\right), 3.92\left(\mathrm{~s}, 3 \mathrm{H}, \mathrm{OCH}_{3}\right), 5.85\left(\mathrm{~s}, 2 \mathrm{H},-\mathrm{O}-\mathrm{C}_{2} \mathrm{O}-\right)$; $6.7 \sim 8.1(\mathrm{~m}, 8 \mathrm{H}$, aromatic protons and $\mathrm{Ar}-\mathrm{CH}=\mathrm{C} \underline{\mathrm{H}}-)$.

Formulation and bioassay. The synergistic activity of all the test compounds was assessed as pyrethrum based emulsions at an insecticide to synergist ratio of $1: 5(\mathrm{w} / \mathrm{w})$. Benzene solvent $(10 \%)$ and Tween-80 emulsifier $(0.2 \%)$ were maintained throughout in the spray emulsions. Two to three week old red flour beetles (Tribolium castaneum
Herbst) reared on wheat flour (free from pesticide) were used as test insects. The tests were conducted in three replications of fifteen insects each, as reported earlier. ${ }^{7)}$ Data were subjected to probit analysis ${ }^{8)}$ and factors of synergism were calculated from the $\mathrm{LC}_{50}$ values. ${ }^{9)}$ Piperonyl butoxide was used as a reference synergist. Purified pyrethrum extract $(20 \%)$ was used for making a stock solution $(4 \%)$ in benzene for biological screening.

Lipophilicity of the test synergists. Lipophilicity of the test compounds was determined by finding out the $R f$ values on reverse phase TLC and then calculating the $R_{\mathrm{M}}$ values by using the equation $R_{\mathrm{M}}=\log [(1 / R f-1)]$. Silica gel plates, after activation for $2 \mathrm{hr}$ at $100^{\circ} \mathrm{C}$, were coated with paraffin in hexane. The coated plates were spotted with the test compound and developed in an acetone-water mixture $(70: 30)$. The positions of all the keto compounds were visualised by spraying with 2,4-dinitrophenyl hydrazine reagent and/or dilute $\mathrm{H}_{2} \mathrm{SO}_{4}$ spray followed by heating. $R f$ values were measured and $R_{\mathrm{M}}$ values were calculated from them. ${ }^{10)}$

\section{REFERENCES}

1) K. C. Gulati and B. S. Parmar, Indian Patent, 119, 536 (1969).

2) E. P. Lichtenstein, T. T. Liang, K. R. Schulz, H. K. Schnoes and G. T. Karter, J. Agric. Food Chem., 22, 658 (1974).

3) S. S. Tomar, V. S. Saxena, M. L. Maheshwari, P. Sarup and S. K. Mukerjee, Indian J. Ent., 40, 113 (1978).

4) S. S. Tomar, M. L. Maheshwari and S. K. Mukerjee, Agric. Biol. Chem., 43, 1479 (1979).

5) S. S. Tomar, M. L. Maheshwari and S. K. Mukerjee, J. Agric. Food Chem., 27, 549 (1979).

6) S. S. Tomar, S. Walia and S. K. Mukerjee, Ind. J. Chem., 19, 792 (1980).

7) S. K. Mukerjee, B. S. Parmar, N. K. Roy and R. P. Singh, Ind. J. Agric. Sci., 43, 516 (1973).

8) D. J. Finney, "Probit Analysis," 3rd Ed., Cambridge University Press, Cambridge, 1971, p. 318.

9) P. R. Chadwick, Pyrethrum Post, 7, 25 (1963).

10) C. B. E. Boyce and B. V. Milborrow, Nature (London), 208, 537 (1965). 